Please do not remove this page

RMIT

UNIVERSITY

\title{
Supercontinuum spatial gap solitons
}

Sukhorukov, Andrey; Neshev, Dragomir; Dreishuh, Alexander; Fischer, Robert; Ha, Sangwoo; Bolger, Jeremy; Bui, Lam

https://researchrepository.rmit.edu.au/esploro/outputs/9921863392201341/filesAndLinks?institution=61RMIT_INST\&index=null

Sukhorukov, A., Neshev, D., Dreishuh, A., Fischer, R., Ha, S., Bolger, J., Bui, L., Krolikowski, W., Eggleton, B., Mitchell, A., Austin, M., \& Kivshar, Y. (2007). Supercontinuum spatial gap solitons. European Conference on Lasers and Electro-Optics, 2007 and the International Quantum Electronics Conference. CLEOE-IQEC 2007., 1-1. https://doi.org/10.1109/CLEOE-IQEC.2007.4386096

Published Version: https://doi.org/10.1109/CLEOE-IQEC.2007.4386096

Repository homepage: https://researchrepository.rmit.edu.au (C) 2007 IEEE

Downloaded On 2023/04/26 19:38:49 +1000 
Citation: Sukhorukov, A, Dreishuh, A, Neshev, D, Fischer, R, Ha, S, Bolger, J, Bui, L, Krolikowski, W, Eggleton, B, Mitchell, A, Austin, M and Kivshar, Y 2007, 'Supercontinuum spatial gap solitons', in European Conference on Lasers and Electro-Optics, 2007 and the International Quantum Electronics Conference. CLEOE-IQEC 2007., Munich, Germany, 17-22 June 2007.

\title{
Supercontinuum spatial gap solitons
}

\author{
Andrey A. Sukhorukov ${ }^{1}$, Dragomir N. Neshev ${ }^{1}$, Alexander Dreischuh ${ }^{1,2}$, \\ Robert Fischer ${ }^{1}$, Sangwoo $\mathrm{Ha}^{1}$, Jeremy Bolger ${ }^{3}$, Lam Bui ${ }^{4}$, Wieslaw Krolikowski ${ }^{1}$, \\ Benjamin J. Eggleton ${ }^{3}$, Arnan Mitchell ${ }^{4}$, Michael W. Austin ${ }^{4}$, and Yuri S. Kivshar ${ }^{1}$ \\ Centre for Ultra-high bandwidth Devices for Optical Systems (CUDOS), \\ ${ }^{1}$ Nonlinear Physics Centre and Laser Physics Centre, \\ RSPhysSE, Australian National University, Canberra, Australia \\ ${ }^{2}$ Department of Quantum Electronics, Faculty of Physics, Sofia University, Bulgaria \\ ${ }^{3}$ School of Physics, University of Sydney, Sydney NSW 2006, Australia \\ ${ }^{4}$ School of Electrical and Computer Engineering, RMTT University, Melbourne, Australia
}

Advances in the generation of light with supercontinuum spectra in photonic-crystal fibers (PCFs) open new possibilities for a wide range of applications. PCFs allow for engineering of the spectral dispersion and confinement of light through the underlying periodicity of their structure. Periodic photonic structures also find applications for spatial beam control, however their use is primarily optimized for beam shaping and deflection in a narrow-frequency range. In this work, we predict theoretically and demonstrate experimentally novel possibilities for active control of supercontinuum light beams in nonlinear waveguide arrays. Whereas different colors are separated in the linear regime due to the strong spectral dispersion, we show that nonlinear interactions enable collective manipulation of amplitude and phase states of all spectral components through the formation of supercontinuum spatial gap solitons.
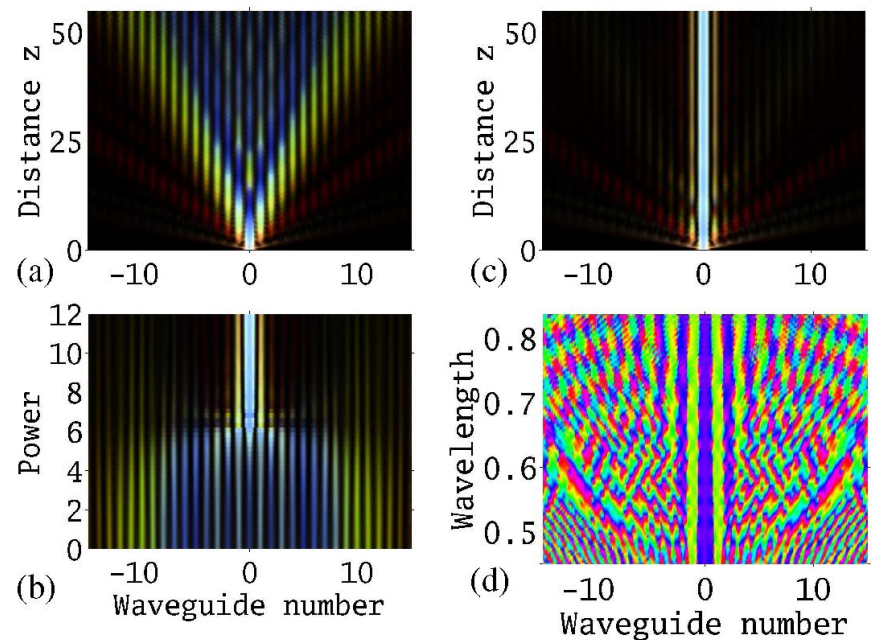

Fig. 1. (a-c) Calculated real-color intensity plots of supercontinuum light beams: (a) linear diffraction inside the waveguide array; (b) output beam profile vs. the input power; (c) soliton formation at high input power. (d) Output phase structure of individual frequency components corresponding to (c)

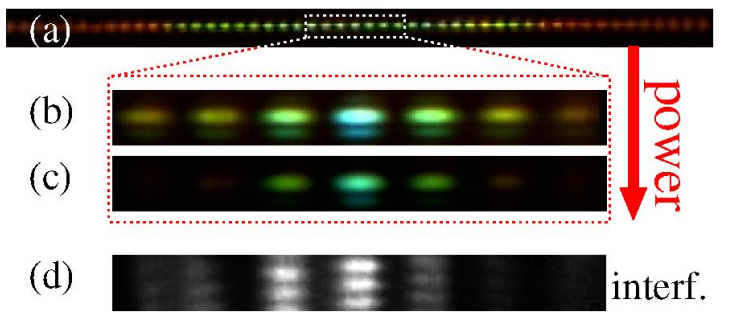

Fig. 2. Experimental observation of supercontinuum gap solitons in the nonlinear waveguide array fabricated in $\mathrm{LiNbO}_{3}$ : (a-c) Real-color CCD camera images of the output beam intensity profile: (a) diffraction profile at low power; (b) nonlinear localization with increasing optical power and (c) formation of a supercontinuum gap soliton. (d) Interferogram of the output beam profile (c) and a reference supercontinuum beam, integrated over all spectral components.

In the linear regime (at low optical powers), each spectral component exhibits typical discrete diffraction where most of the light is concentrated into the wings of the beam rather than in its center. Spectral dispersion leads to the separation of various colors among different channels of the waveguide array, as shown in Figs. 1(a) and 2(a). We demonstrate that collective manipulation of all colors becomes possible when spectral dispersion is compensated by nonlinear incoherent interactions between spectral components in photorefractive media through the optically-induced decrease of the optical refractive index. Although such a negative nonlinear response would result in self-defocusing and accelerated beam broadening in bulk media, the supercontinuum beam experiences sharp transition to self-trapping in a periodic waveguide array above a critical power level [Fig. 1(b)]. The formation of such self-localized gap solitons [Fig. 1(c) and 2(c)] becomes possible due to localization in the photonic bandgap ${ }^{1}$, when the dominating spectral components in the adjacent waveguides are out-of-phase [Fig. 1(d) and 2(d)], demonstrating nontrivial phase control of supercontinuum radiation. This represents a uniquely different phenomenon compared to the theoretically studied white-light lattice solitons in selffocusing media ${ }^{2}$.

\section{References}

1. K. Motzek, A. A. Sukhorukov, and Yu. S. Kivshar, Opt. Express 14, 9873 (2006).

2. R. Pezer, H. Buljan, G. Bartal, M. Segev, and J. W. Fleischer, Phys. Rev. E 73, 056608 (2006). 